\title{
Vigilance in Nurses Working in Intensive Care Units
}

\author{
Mehdi Ajri-Khameslou, Mojgan Najafi, Mansoureh Karimollahi* \\ Nursing Department, School of Nursing and Midwifery, Ardabil University of Medical Sciences, Ardabil, Iran \\ Email: ^m.karimollahi@arums.ac.ir, ${ }^{\star}$ karimollahi@gmail.com
}

How to cite this paper: Ajri-Khameslou, M., Najafi, M. and Karimollahi, M. (2021) Vigilance in Nurses Working in Intensive Care Units. Open Journal of Nursing, 11, 715-727.

https://doi.org/10.4236/ojn.2021.119061

Received: August 11, 2021

Accepted: September 14, 2021

Published: September 17, 2021

Copyright $\odot 2021$ by author(s) and Scientific Research Publishing Inc. This work is licensed under the Creative Commons Attribution International License (CC BY 4.0).

http://creativecommons.org/licenses/by/4.0/

\begin{abstract}
Background and Aim: Vigilance is an essential element in intensive care nursing. This study was conducted to determine nursing vigilance in nurses working in the intensive care units of educational and medical centers in Ardabil, Iran. Methods: This was a cross-sectional descriptive-analytical study with 192 ICU nurses as the participants. The data were obtained through questionnaires regarding demographic characteristics and nursing vigilance. SPSS software version 24 was used for the statistical analysis. Results: The mean total vigilance score was $3.86 \pm 0.23$ of 5 . The mean scores of the timely diagnosis of changes, pattern recognition, and clinical decision-making subscales were $4.07 \pm 0.26,04.04 \pm 0.41$, and $3.44 \pm 0.25$, respectively. No significant relationships were observed between the total or subscale vigilance scores and other demographic characteristics. Conclusion: We assessed ICU nurses' vigilance behaviors and found that their mean vigilance score was higher than the expected average, indicating our participants had a high level of clinical vigilance. These results suggest a need for effective educational interventions to boost clinical decision-making skills in ICU nurses, especially younger nurses.
\end{abstract}

\section{Keywords}

Nursing Vigilance, Intensive Care Unit, Nurse, Clinical Decision-Making

\section{Introduction}

Patients hospitalized in intensive care units (ICUs) are vulnerable to health threats given their special physiological status, pharmaceutical interventions, and being supervised by multiple caregivers [1]. Due to the severity of their illnesses, these patients lack the flexibility and ability to cope with the consequences of medical errors [2], which can be life-threatening [1]. Since nurses 
working in an ICU provide a major part of the health care for these patients, they must be vigilant enough to provide safe services [3] [4] and upgrade patient safety [5].

Vigilance is a largely invisible but essential element of intensive care nursing [4]. The term "vigilance" is derived from the old French word "vigilia", meaning a state or practice of being careful about potential dangers or problems [6]. Loeb and Alluisi [7] introduced vigilance in their signal recognition theory and characterized vigilance as a search for signals when the events construed by individuals as important occur in a noisy background. The literature related to stress and its coping strategies defines vigilance as a reaction expressed through thoughts and actions in response to perceived threats [8].

In terms of health care services, vigilance refers to nurses anticipating, monitoring, and acting to minimize health threats and ensure patient safety during care [6]. Vigilance is defined as an accurate and adequate focus and high physical and mental readiness to recognize and respond to danger [9]. Professional vigilance in nursing, as the art of watching, is the essence of nursing and a prerequisite for informed nursing practice [9] [10].

Maintaining sustained and high-level vigilance in nursing, especially intensive care nursing, requires constant watchfulness in an environment of alarms, contact lights, and telephones, as well as the ability to act immediately to ensure patient safety [11]. Krause et al. [12] introduced a model of sustained vigilance based on the idea of the vigilance exhaustion experienced by law enforcement personnel. Their model suggests there is a multilateral relationship between the concept of sustained vigilance and three contributing factors-attention, situational vigilance, and decision making; thus, the absence of any of these factors weakens vigilance [12]. Experimental studies on vigilance were first conducted in the defense industry to identify how to upgrade radar operators to enable the detection of signals from approaching enemies [13]. In Kooken et al.'s [8] study, nurses in an oncology ward acknowledged applying vigilance to counteract potential health threats and maintain patient safety and agreed vigilance was part of their routine work. Vigilance coordinated with their professional performance, helping them remain aware of any health-threatening factors [13]; it thus can be understood as a force that enables nurses to guard patient safety [14]. Geib [15] introduced the concept of nursing vigilance using three subcategories: 1) Early detection of patient health changes; 2) Knowing patients' general conditions to allow pattern recognition; 3) Clinical decision-making.

Low vigilance while performing nursing procedures may lead nurses to perpetuate a mistake or turn a potential error into an actual one [16]. According to Thomson, Smilck, and Burns [17], health care providers with low vigilance are more likely to make medical errors. Factors such as drowsiness, fatigue, mental preoccupation, low work experience, prolonged shift time, excessive working hours per week, and inadequate rest intervals can significantly affect nursing vigilance and patient safety [18]. Although research in this area is limited, studies have shown a clear relationship between prolonged working time and reduced 
nursing vigilance [4]. Rogers et al. [3] showed that nurses who worked more than 12 consecutive hours or more than 40 hours per week were at higher risk of making errors. In Scott et al.'s study, most nurses stated that they suffered from relatively severe fatigue, a remarkable reduction in sleep hours, and daytime drowsiness and confirmed that these factors would affect their vigilance. Furthermore, long work shifts lead to the loss of situational vigilance and creativity and errors in problem-solving and decision-making, which reduce alertness and compromise patient safety [2].

A few studies on nurses' mental and behavioral vigilance have been conducted from the nurses' perspective. Nursing vigilance is particularly important for providing safe health services, protecting patients from medical errors, preventing deterioration in patients' clinical conditions, and avoiding the imposition of additional costs on both the health system and patients' families. Considering how these factors and individual and environmental factors impact nurses' vigilance, there is a need for comprehensive studies in this area. Therefore, our goal was to identify the vigilance of nurses working in the ICUs of educational and medical centers in Ardabil, Iran.

\section{Method}

This cross-sectional descriptive-analytical study was conducted in 2019. The study population included nurses working in the ICUs of educational and medical centers in Ardabil, Iran. Inclusion criteria were the willingness of nurses to participate in the study and at least six months of work experience in the ICU. The exclusion criterion was the incomplete completion of the questionnaire. Due to the small size of the study population, we used the consensus sampling method. Therefore, all nurses working in the ICUs of Ardabil educational and medical centers were included $(n=216)$. Among those nurses, $192(88.8 \%)$ fully completed the questionnaire, and these data were analyzed.

The data were collected using a two-part questionnaire. The first part addressed the nurses' demographic information, including age, sex, marital status, education, work shift, work experience, and overtime hours per month. The second part was the Nursing Vigilance Scale (NVS) (appendix), which consisted of 40 items and three subscales: Timely identification of patient change (TIPC) with 10 items, knowing the patient to allow pattern recognition (KPPR) with 13 items, and clinical decision-making (CDM) with 17 items. Each question was scored based on a 5-point Likert scale from 1 (never) to 5 (always), and the scores ranged from 40 to 200 [15]. Nine questions had an inverse scoring system. The instrument validity was evaluated by having 10 faculty members of the Nursing School of Ardabil, and 5 senior clinical experts provide their comments about the questionnaire's face validity. The recommended corrections were then applied. To quantitatively determine content validity, two indices-the content validity ratio (CVR) and content validity index (CVI) - were calculated. To determine the CVR, we obtained experts' opinions about the need for each ques- 
tion. The CVR obtained for each statement was higher than the value specified in the Lawshe table (0.49). Therefore, in terms of necessity, all the questions with scores between 0.6 and 1 had acceptable validity. Regarding the simplicity, clarity, and relevance of each question, the CVI index obtained for each statement was higher than 0.79 (the range of 0.8 to 1 ) and acceptable. The instrument reliability was calculated based on internal coherence using Cronbach's alpha coefficient, which indicated acceptable reliability $(\alpha=0.74)$.

The study was conducted in the ICUs of trauma, neurology, internal surgery, and pediatric hospitals. A productivity plan was implemented in all of these hospitals, meaning that no nurses were allowed to work more than 12 consecutive hours. Additionally, each nurse took care of at least two patients.

For statistical analysis, the data were entered into SPSS (version 24), which produced descriptive statistics (frequency, percentage, mean, median, standard deviation, minimum, and maximum). Using a significance level of $\mathrm{p}<0.05$, independent sample $t$-tests and ANOVA were applied to compare vigilance scores according to demographic characteristics and other variables.

\section{Results}

This study included 192 ICU nurse participants. The mean age of the nurses was $33.76 \pm 5.69$ years, $94.4 \%$ of them were women, and $77.6 \%$ were married. Most participants (88\%) worked in rotational shifts. The average work experience was $9.22 \pm 5.56$ years, and most of the participants had $1-10$ years of work experience. The mean overtime hours per month was $72.23 \pm 28.10$ (median $=70$ ), and most of the nurses had from 50 to 100 overtime hours per month. Overall, $96.4 \%$ and $3.6 \%$ of the nurses had bachelors' and master's degrees, respectively (Table 1).

The mean total vigilance score obtained was $3.86 \pm 0.23$, while the mean scores of the TIPC, KPPR, and CDM subscales were $4.07 \pm 0.26,4.04 \pm 0.41$, and $3.44 \pm 0.25$, respectively (Table 2 ).

According to our results, higher mean scores were related to the TIPC subscale, while lower scores were observed for the CDM subscale and negative expressions with inverse scoring (Table 3 ).

The results regarding demographic variables and nursing vigilance showed a statistically significant relationship between age and the CDM subscale; the mean CDM score was higher in older age groups (41 - 50 years) compared to younger age groups $(P=0.022)$. However, the total score and the other two subscales were not significantly associated with participant age. There were no significant relationships between other demographic variables and the total or subscale vigilance scores.

On the TIPC subscale, male nurses achieved a higher score $(4.18 \pm 0.20)$ than females $(4.07 \pm 0.26)$, while females attained a better score $(4.04 \pm 0.41)$ than males (3.96 \pm 0.35$)$ in the KPPR subscale; however, this difference was not statistically significant using an unpaired t-test. Additionally, there was no statistically 
significant relationship between marital status and either the total or subscale vigilance scores.

The total vigilance score was higher in nurses working in fixed morning shifts $(3.90 \pm 0.16)$ than in rotational shifts $(3.86 \pm 0.24)$. However, the unpaired t-test revealed no significant relationship between the work shift type and total or subscale vigilance scores. The total vigilance score of nurses with master's degrees was $3.97 \pm 0.21$, which was higher than the score of those with bachelor's degrees $(3.86 \pm 0.23)$. Nurses with higher education also attained higher scores on the TIPC, KPPR, and CDM subscales; however, unpaired t-tests showed that these differences were not statistically significant.

Table 1. Demographic characteristics of nurses working in intensive care units.

\begin{tabular}{|c|c|c|c|}
\hline Demographic Characteristics & categories & Number $(N)$ & Percent (\%) \\
\hline \multirow{3}{*}{ Age (years) } & $23-40$ & 68 & 35.4 \\
\hline & $31-40$ & 100 & 52.1 \\
\hline & $41-50$ & 24 & 12.5 \\
\hline \multirow{2}{*}{$\operatorname{sex}$} & Male & 5 & 2.6 \\
\hline & Female & 187 & 97.4 \\
\hline \multirow{2}{*}{ Marital status } & Single & 43 & 22.4 \\
\hline & Married & 149 & 77.6 \\
\hline \multirow{5}{*}{ Work experience (years) } & $1-5$ & 60 & 31.3 \\
\hline & $6-10$ & 60 & 31.3 \\
\hline & $11-15$ & 43 & 22.4 \\
\hline & $16-20$ & 23 & 12 \\
\hline & $21-25$ & 6 & 3.1 \\
\hline \multirow{2}{*}{ Working shift } & Constant & 23 & 12 \\
\hline & Circular & 169 & 88 \\
\hline \multirow{3}{*}{ Overtime work (hours) } & $0-50$ & 47 & 24.5 \\
\hline & $51-100$ & 125 & 65.1 \\
\hline & $101-150$ & 20 & 10.4 \\
\hline \multirow{2}{*}{ Education } & Bachelors & 185 & 96.4 \\
\hline & Master & 7 & 3.6 \\
\hline
\end{tabular}

Table 2. The mean and standard deviation of the total and subscale scores of nursing vigilance in the studied population.

\begin{tabular}{ccccc}
\hline Subscales & Mean & SD & Min & Max \\
\hline Timely Identification of Patient Change (TIPC) & 4.07 & 0.26 & 3.20 & 4.60 \\
Knowing the Patient for Pattern Recognition (KPPR) & 4.04 & 0.41 & 2.77 & 5 \\
Clinical Decision Making (CDM) & 4.44 & 0.25 & 2.76 & 4.18 \\
Total vigilance & 3.86 & 0.23 & 3.28 & 4.55 \\
\hline
\end{tabular}


Table 3. The mean and standard deviation of the questions with the highest and lowest scores.

\begin{tabular}{|c|c|c|c|c|}
\hline Score & Number of $Q$ & Statements & Mean & $S D$ \\
\hline \multirow{6}{*}{ Highest } & 16 (TIPC) & I monitor symptoms to predict changes in patients' conditions. & 4.56 & 0.59 \\
\hline & 26 (KPPR) & I would like to be available to meet patients' needs. & 4.55 & 0.63 \\
\hline & 14 (TIPC) & I constantly check patients' hemodynamic indicators on the monitoring system. & 4.53 & 0.69 \\
\hline & 12 (TIPC) & I try to be prepared to respond to changes in patients' conditions. & 4.52 & 0.57 \\
\hline & 29 (KPPR) & I explain to patients about provided nursing procedures. & 4.49 & 0.63 \\
\hline & 9 (TIPC) & I check patients' test results at specific times to monitor their current condition. & 4.47 & 0.66 \\
\hline \multirow{5}{*}{ Lowest } & 39 (TIPC) & I check patients' test results once per shift. & 1.64 & 0.76 \\
\hline & $25(\mathrm{CDM})$ & My past experiences have urged me to consider the pros and cons of making decisions about patients. & 1.73 & 0.71 \\
\hline & $10(\mathrm{CDM})$ & I need to reach a consensus with my colleagues when making decisions. & 1.85 & 0.8 \\
\hline & $22(\mathrm{CDM})$ & I consider my colleagues' reactions when I think about my options. & 2.27 & 0.77 \\
\hline & $40(\mathrm{CDM})$ & I am randomly looking for new information. & 2.66 & 0.95 \\
\hline
\end{tabular}

TIPC: Timely Identification of Patient Change; KPPR: Knowing the Patient for Pattern Recognition; CDM: Clinical Decision Making.

Although compared to the other groups, participants with 16 - 20 years of working experience received higher total vigilance scores (3.95 \pm 0.21$)$, as well as better scores in the TIPC (4.13), KPPR (4.14), and CDM (3.50) subscales, an ANOVA was conducted, which showed that these differences were not statistically significant.

Finally, nurses who worked more than 100 hours of overtime per month had higher scores in total and in the TIPC, KPPR, and CDM subscales; nevertheless, the differences between the groups were not statistically significant.

\section{Discussion}

This was a descriptive cross-sectional study conducted to determine the vigilance of nurses working in ICUs. The total vigilance score was higher than the expected average, indicating that the nurses had a relatively high level of vigilance in clinical practice. Geib [15] also reported a vigilance level higher than the expected range in her study. Other studies using different scales to assess nursing vigilance and patient safety have also reported high vigilance levels in the performance of daily nursing care. For example, Vahidi et al. [6] conducted interviews with nurses working in psychiatric wards and showed that nurses were able to predict events and take appropriate preventive measures by cognitively evaluating patients through the assimilation of data from their observations and available risk assessment patterns. Additionally, O'Brien et al. [19] explained that nurses in the recovery room reduced risk and ensured patient safety using team and instant coordination strategies. In Ajri et al.'s [20] study, nurses noticed and tried to prevent mistakes by monitoring, questioning, and self-reporting errors.

Of the subscales, the CDM showed the lowest score, consistent with Geib [15]. According to Maharmeh et al. [21], the difficult and complicated statuses of the 
health of patients in the ICU highlight the complexity and importance of CDM by nurses. Patients' unstable and rapidly changing conditions require nurses to make decisions within a limited time. This study's findings suggest it can be argued that nurses working in ICUs should deal with at least two patients with different characteristics and needs. On the other hand, making independent decisions for each patient can be challenging for these nurses. Accordingly, the nurses participating in this study obtained lower scores in their responses to the statements under the CDM subscale that had inverse scoring. Overall, considering the impact of CDM on patient care and recovery, there is a need to implement effective educational interventions to improve the CDM skills of nurses working in ICUs.

In the CDM subscale, the lowest average scores were related to the phrases, "It is important to me to reach consensus with my colleagues when making decisions" and "I consider my colleagues' reactions when I think about my options". Most nurses replied to these phrases with "always" or "usually" instead of "never" as the answer with the highest score. This indicates the participants' perceived interaction with other nurses as a best practice in decision-making, while nurses should play a role as an individual fully informed of their patient's condition and the main decision-maker, obviating the roles of others in this process. One of the reasons for this observation could be that these wards employed nurses with little experience. It may also indicate that nursing education and internship periods have not significantly improved nurses' confidence and independence in decision-making, which are among the main professional nursing competency components. Since taking more responsibility requires that professional nurses attain independence in their activities, including decision-making, new educational methods are needed to empower nurses' critical thinking skills and boost their self-confidence. The curriculum should be modified to help students know themselves and develop independent behaviors and make them familiar with professional rules and duties. Finally, managers must support nurses to promote their independence in decision-making.

Another phrase, "My past experiences have urged me to consider the pros and cons of making decisions about patients", also attained a low score. The expected reply to this phrase was "never"; however, most participants chose either "always" or "usually". This was consistent with the findings of Maharmeh et al. and Clark et al. [21] [22], who declared that clinical experience was one of the main determinants of nurses' decision making. However, this study suggests that the participants only exploited their experience as a tool for critical thinking and clinical reasoning, setting aside the knowledge and skills learned in nursing school.

Our participants demonstrated the highest score in the TIPC subscale, which is consistent with the findings in Geib [15]. The highest scores were related to the phrases, "I monitor symptoms to predict changes in patients' condition", "I constantly check patients' hemodynamic indicators on the monitoring system", "I try to be prepared to respond to changes in patients' conditions" and "I check 
patients' test results at specific times to monitor their current condition." These results demonstrate the importance of careful monitoring and immediate responses by nurses. According to Kagan, Nightingale was the first to assert the concepts of vigilance and monitoring in 1850 [23], and defined monitoring as a regular assessment, in addition to analyzing patient information and providing prompt responses to events. Consistent with our observation, Meyer and Lavin [9] specified that being skilled at the main vigilance components is necessary for early detection of patient problems, recognizing the causes of deterioration of patient conditions, and timely responses to the consequences. On the other hand, Hillman et al. [24] found that $29 \%$ of patients with cardiac arrest had serious physiological instabilities 8 hours before death, and one-third of them experienced these variations 48 hours before death. The most common physiological instabilities were hypotension and tachypnea. Buist et al. [25] reported physiological instabilities before preventable death, such as drops in blood pressure and oxygen saturation and significant changes in heart and respiration rates, were the predominant predictors of mortality; failure to identify these alternations in a timely manner significantly contributed to the death. Alexa et al. [26] examined the factors affecting sepsis diagnosis and failure to resuscitate (FTR) among 57 nurses working in an oncology ICU and reported that FTR mainly occurred after nurses failed to monitor, detect, and correctly perceive patient data. The results of these studies, however, are inconsistent with our findings of a high score in detecting changes in patient conditions. This discrepancy may be because the aforementioned studies were retrospective and applied objective measurement tools, unlike the current study which applied a mental tool.

In the present study, a significant relationship was observed between the CDM subscale and participant age. Geib [15] also reported such a relationship; however, it was statistically insignificant. In Parasuraman and Giambra's [27] review of 13 studies, half the studies indicated that CDM was related to age and delayed by advancing age. Likewise, in Cornelia et al. [28], the reaction time of the youngest age group (20 - 29 years) was significantly shorter than that of participants aged 60 - 69 or $70-79$ years. These findings indicate that age plays an important role in promoting CDM in nurses. Therefore, implementing educational and practical interventions to improve the ability of young nurses to make informed decisions may potentially impact their caring experience and improve the outcomes of health care services.

As with all studies, this study has some limitations; these include the small sample size, the low ratio of male nurses, and the low number of nurses with master's degrees. Therefore, care should be taken in generalizing our findings to all nurses. In this study, we used a mental tool and self-reporting to assess vigilance; however, it is also important to examine nurses' vigilance during patient care. Therefore, it is advisable to utilize a combination of both subjective and objective measurement scales to assess vigilance and its relationship with other variables. 


\section{Conclusion}

This study's findings showed a higher than expected mean vigilance score in our participants, which indicated their high level of vigilance in clinical practice. However, the participants exhibited a low score in the CDM subscale, which was significantly associated with participant age. Therefore, our results suggest that effective educational interventions be implemented to strengthen CDM skills in nurses working in ICUs, especially younger nurses. In our study, vigilance was not significantly related to demographic features. Overall, our observations highlight the importance of vigilance in ICU nurses. More research is needed to establish nursing vigilance as a determining factor in patient outcomes and an essential component of professional nursing practice.

\section{Ethics Approval and Consent to Participate}

Ethical approval was provided by the Ethics committee of Ardabil University of Medical Sciences (IR.ARUMS.REC.1395.114). Written informed consent was obtained from all subjects.

\section{Funding}

This project was funded by the research deputy of Ardabil University of Medical Sciences.

\section{Authors' Contributions}

M.A. M.J., M.K. conceptualized and designed the study and acquired the data. M.A.M.J., M.K. analyzed the data and drafted the manuscript. The manuscript is revised critically for the important intellectual content and all authors read and approved the final manuscript.

\section{Availability of Data and Materials}

Data will be made available upon reasonable request to researchers who provide a methodologically sound proposal, after approval by the study authors and with a signed data access agreement. Questions about data are handled by the corresponding author.

\section{Consent for Publication}

The manuscript is revised critically for the important intellectual content and all authors read and approved the final manuscript.

\section{Acknowledgements}

The authors would like to sincerely thank all those who collaborated in this research, especially the deputy of research of the Nursing and Midwifery School. We also thank the staff of educational and medical centers and the nurses who participated in this project. 


\section{Conflicts of Interest}

The authors declare no conflicts of interest regarding the publication of this paper.

\section{References}

[1] Urden, L.D., Stacy, K.M. and Lough, M.E. (2017) Critical Care Nursing-E-Book: Diagnosis and Management. Mosby, Maryland Heights.

[2] Scott, L.D., Rogers, A.E., Hwang, W.-T. and Zhang, Y. (2006) Effects of Critical Care Nurses' Work Hours on Vigilance and Patients' Safety. American Journal of Critical Care, 15, 30-37. https://doi.org/10.4037/ajcc2006.15.1.30

[3] Rogers, A.E., Hwang, W.-T., Scott, L.D., Aiken, L.H. and Dinges, D.F. (2004) The Working Hours of Hospital Staff Nurses and Patient Safety. Health Affairs, 23, 202-212. https://doi.org/10.1377/hlthaff.23.4.202

[4] Balas, M.C., Casey, C.M., Scott, L.D. and Rogers, A.E. (2007) Maintain Vigilance in the ICU. Nursing Critical Care, 2, 38-44. https://doi.org/10.1097/01.CCN.0000281592.23608.20

[5] Adenusi, B. (2018) The Importance of Vigilance in Promoting Patient Safety in Nurse Anesthesia Practice: A Correlational Study. New Mexico State University, Las Cruces.

[6] Vahidi, M., Ebrahimi, H., Areshtanab, H.N., Jafarabadi, M.A., West, S., Foong, A., et al. (2019) Providing a Safe Environment in a Psychiatric Ward: Nurses and Vigilance in Iran. Issues in Mental Health Nursing, 40, 65-72. https://doi.org/10.1080/01612840.2018.1463326

[7] Loeb, M. and Alluisi, E.A. (1984) Theories of Vigilance. In: Warm, J.S., Sustained Attention in Human Performance, Wiley, New York, 179-205.

[8] Kooken, W.C. (2008) Vigilance Experiences: Cancer Patients, Families, and Nurses. Ph.D. Thesis, Indiana University, Bloomington.

[9] Meyer, G. and Lavin, M.A. (2005) Vigilance: The Essence of Nursing. Online Journal of Issues in Nursing, 10, 38-51.

[10] Surani, S., Hesselbacher, S., Guntupalli, B., Surani, S. and Subramanian, S. (2015) Sleep Quality and Vigilance Differ among Inpatient Nurses Based on the Unit Setting and Shift Worked. Journal of Patient Safety, 11, 215-220. https://doi.org/10.1097/PTS.0000000000000089

[11] Morelock, S.G. (2014) Sustained Vigilance, Errors, and Job Satisfaction in a Population of Critical Care and Emergency Department Nurses. Dctoral Thesis, The University of Texas at Tyler, Tyler, Paper No. 40.

[12] Krause, M. (2012) Vigilance Fatigue in Policing: A Critical Threat to Public Safety and Officer Well-Being. FBI Law Enforcement Bulletin, 81, Article No. 3.

[13] Kooken, W.C. and Haase, J.E. (2014) A Big Word for Something We do All the Time: Oncology Nurses Lived Experience of Vigilance. Cancer Nursing, 37, E15-E24. https://doi.org/10.1097/NCC.0000000000000113

[14] Johansson, L. and Wiklund-Gustin, L. (2016) The Multifaceted Vigilance-Nurses' Experiences of Caring Encounters with Patients Suffering from Substance Use Disorder. Scandinavian Journal of Caring Sciences, 30, 303-311. https://doi.org/10.1111/scs.12244

[15] Geib, K.M. (2003) The Relationships among Nursing Vigilance by Nurses, Patient Satisfaction with Nursing Vigilance, and Patient Length of Stay in a Surgical Car- 
diac Care Unit. Podium Presentation the Biennial Meeting of the International Neuman Systems Model Symposium, Willow Grove, 2003, 248 p.

[16] Emrich, L. (2004) The Incidence of Nursing Non-Vigilance and Diminished Nursing Vigilance in Selected Cases Investigated by the Ohio Board of Nursing. Capital University, Columbus, Ohio.

[17] Thomson, D.R., Smilek, D. and Besner, D. (2015) Reducing the Vigilance Decrement: The Effects of Perceptual Variability. Consciousness and Cognition, 33, 386-397. https://doi.org/10.1016/j.concog.2015.02.010

[18] Sagah Zadeh, R., Shepley, M., Sadatsafavi, H., Owora, A.H. and Krieger, A.C. (2018) Alert Workplace from Healthcare Workers' Perspective: Behavioral and Environmental Strategies to Improve Vigilance and Alertness in Healthcare Settings. Herd: Health Environments Research \& Design Journal, 11, 72-88. https://doi.org/10.1177\%2F1937586717729349

[19] O’Brien, B., Andrews, T. and Savage, E. (2018) Anticipatory Vigilance: A Grounded Theory Study of Minimizing Risk within the Perioperative Setting. Journal of Clinical Nursing, 27, 247-256. https://doi.org/10.1111/jocn.13881

[20] Ajri-khameslou, M., Aliyari, S., Pishgooie, A., Jafari-Golestan, N. and Afshar, P.F. (2021) Concept of Error and Nature of Nursing Error Detectors in Military Hospitals: A Qualitative Content Analysis. BMJ Military Health, 167, 48-52.

[21] Maharmeh, M., Alasad, J., Salami, I., Saleh, Z. and Darawad, M. (2016) Clinical Decision-Making among Critical Care Nurses: A Qualitative Study. Health, 8, 1807-1819. https://doi.org/10.4236/health.2016.815173

[22] King, L. and Clark, J.M. (2002) Intuition and the Development of Expertise in Surgical Ward and Intensive Care Nurses. Journal of Advanced Nursing, 37, 322-329. https://doi.org/10.1046/j.1365-2648.2002.02105.x

[23] Kagan, R.N. (2016) An Evidence-Based Educational Intervention to Improve Nursing Staff's Critical Thinking and Decision-Making Skills. Doctoral Thesis, Walden University, Minneapolis.

[24] Hillman, K., Bristow, P., Chey, T., Daffurn, K., Jacques, T., Norman, S., et al. (2001) Antecedents to Hospital Deaths. Internal Medicine Journal, 31, 343-348. https://doi.org/10.1046/j.1445-5994.2001.00077.x

[25] Buist, M., Bernard, S., Nguyen, T.V., Moore, G. and Anderson, J. (2004) Association between Clinically Abnormal Observations and Subsequent In-Hospital Mortality: A Prospective Study. Resuscitation, 62, 137-141. https://doi.org/10.1016/j.resuscitation.2004.03.005

[26] Doig, A., Baraki, K. and Drews, F. (2013) Cognitive Factors Associated with Nurses' Successful Detection of Sepsis versus Failure to Rescue. Proceedings of the International Symposium on Human Factors and Ergonomics in Health Care, 2, 131-134. https://doi.org/10.1177\%2F2327857913021025

[27] Parasuraman, R. and Giambra, L. (1991) Skill Development in Vigilance: Effects of Event Rate and Age. Psychology and Aging, 6, 155-169.

https://doi.apa.org/doi/10.1037/0882-7974.6.2.155

[28] Sauter, C., Danker-Hopfe, H., Loretz, E., Zeitlhofer, J., Geisler, P. and Popp, R. (2013) The Assessment of Vigilance: Normative Data on the Siesta Sustained Attention Test. Sleep Medicine, 14, 542-548. https://doi.org/10.1016/j.sleep.2013.01.011 


\section{Abbreviations}

ICU: intensive care unit;

CVI: content validity index;

CVR: content validity ratio;

CDM: clinical decision-making;

TIPC: timely identification of patient change;

NVS: Nursing Vigilance Scale;

KPPR: knowing the patient to allow pattern recognition.

\section{Appendix}

1) If the clinical decision is vital and there is no time pressure, I do a thorough search for alternative replacement.

2) If I had only one patient, I would care with continuous attendance.

3) The situational factors determine the number of alternatives I find before decision-making.

4) I continuously supervise the parameters of patient monitor.

5) I do not check new information while making a decision because it is not worth.

6) I supervise the vital symptoms for prognosis of changes in patient's condition.

7) I randomly check the alternatives while making a decision because it is more efficient.

8) I do my best to obtain more information for decision-making.

9) I occasionally investigate the current results of lab tests.

10) It is important for me to reach a consensus among colleagues.

11) I occasionally investigate the current results of patient's diagnostic studies.

12) I try to be prepared to respond to changes in patient's condition.

13) I solve problems or make a decision using the available information without any consultation.

14) I obtain information about patient's pathology results before operation.

15) I obtain information about patient's family or every other important condition.

16) I obtain information about patient's mental status about operation.

17) I make a mental list of alternatives before making a decision.

18) While investigating consequences of alternatives I may choose, I usually think about: "if I had done this ..."

19) I obtain information about patient's other health problems.

20) I obtain information about patient's history of work and social activities.

21) I consider patient as source of information.

22) When I think about possible choices I could make, I consider how my colleagues would judge me.

23) I obtain information about patient's spiritual and cultural considerations for nursing care.

24) It is important for me to speak with patients about their comfort.

25) My past experience determines how I should pay attention to dangers and benefits of decision-making about patient. 


\section{Continued}

26) I like to be available on patient's request.

27) While investigating consequences of alternatives I may choose, I am aware of positive results for my patient.

28) When I assess an important clinical decision, I make a list of positive and negative consequences.

29) I explain nursing actions to the patient while caring.

30) While making clinic decision, I consider the organizational priorities and standards.

31) If the situation demands, I allow others interfere with decision-making.

32) I know how the patient thinks and feels about interpretation of changes in patient's condition.

33) Finding about the patient's goals is a common part of my clinic decision-making.

34) I want to know patient's past experiences about the disease to interpret the patient's behaviors.

35) The patient's values should be compatible with mine to be able to make a good decision.

36) I act like a detective while caring the patient.

37) If my colleague recommends an alternative for a clinic decision, I accept it instead of searching for other alternatives.

38) When the patient's condition changes, I know if I should interfere or wait and observe.

39) I check lab results once in each shift.

40) I randomly look for new information.

41) I immediately respond to patient's expressed worries. 